\title{
Targeted therapy in HER2-positive breast cancer (Review)
}

\author{
SHU GUANG LI and LI LI \\ Department of Chemotherapy, Cancer Center, Qilu Hospital of Shandong University, Jinan, \\ Shandong 250012, P.R. China
}

Received March 3, 2013; Accepted April 4, 2013

DOI: $10.3892 /$ br.2013.95

\begin{abstract}
Treatment options for breast cancer vary based on tumor surface markers and clinical factors, including cytotoxic chemotherapy, hormonal therapy, biological therapy or a combination thereof. An important molecular determinant of therapy is the human epidermal growth factor receptor 2 (HER2) positivity of the tumor, which has been identified in $20-25 \%$ of breast cancers and is a prognostic marker of poor outcome. The advent of HER2-targeted therapies has significantly improved the survival of patients with HER2-positive breast cancer. This review focuses on current HER2-targeted therapeutic options for patients with HER2-positive breast cancer, including monoclonal antibodies and tyrosine kinase inhibitors (TKIs).
\end{abstract}

\section{Contents}

1. Introduction

2. Monoclonal antibodies

3. Tyrosine kinase inhibitors

4. Conclusion

\section{Introduction}

Breast cancer is the most frequently diagnosed cancer and one of the major causes of mortality in females worldwide. Breast cancer is also one of the most investigated diseases and its management has progressed rapidly into the molecular era. The current therapies have merged clinical, pathological and molecular understanding to improve outcomes, resulting in a decrease in mortality.

Correspondence to: Dr $\mathrm{Li} \mathrm{Li}$, Department of Chemotherapy, Cancer Center, Qilu Hospital of Shandong University, 107 West Wenhua Road, Jinan, Shandong 250012, P.R. China

E-mail: 1ili_5060@yahoo.com.cn

Key words: breast cancer, human epidermal growth factor receptor 2, targeted therapy, monoclonal antibodies, tyrosine kinase inhibitors
One of the major challenges in breast cancer treatment stems from the fact that it is a heterogeneous disease comprising at least five subtypes (1). It has become evident that $20-25 \%$ of breast cancers are classified as human epidermal growth factor receptor 2 (HER2)-positive, which denotes an aggressive phenotype resulting in reduced disease-free and overall survival compared with other breast cancer subtypes (2,3). HER2 belongs to the human epidermal grow th factor receptor (EGFR) family, which includes the closely related receptors HER1 (or EGFR), HER2, HER3 and HER4, also known as the HER family. HER receptors are transmembrane glycoproteins containing an extracellular ligand-binding domain and an intracellular receptor tyrosine kinase (TK) domain, bothe of which are important in tumor development via their effect on cell proliferation, migration, angiogenesis and anti-apoptosis (4). Although the subtypes of HER receptors have distinct extracellular ligand-binding domains, they share a similar TK domain (5). Ligand binding results in receptor homo- or hetero-dimerization. HER2 has no known ligand and is activated through the heterotypic interaction of its extracellular domain (ECD) with that of other EGFR receptors (6). It is also the preferred dimerization partner within the EGFR family (7). Within dimers, the interactions between the intracellular domains of the receptors lead to autophosphorylation of the tyrosine kinase, allowing for subsequent signal transduction which is associated with cell proliferation, apoptosis, angiogenesis and metastasis (4). In addition to being a reliable biomarker, HER2 is a validated therapeutic target. Treatment specifically targeted at HER2 has dramatically improved survival during the past decade in patients with HER2-positive breast cancer. This review focuses on current treatments for patients with HER2-positive breast cancer, including monoclonal antibodies and TK inhibitors (TKIs) which have markedly improved the natural history of HER2-positive breast cancer.

\section{Monoclonal antibodies}

Trastuzumab. Trastuzumab (Herceptin; Genentech/Roche, SouthSanFrancisco,CA,USA), thefirstavailableHER2-targeted therapy, is a humanized murine $\operatorname{IgG}$ monoclonal antibody that binds to the HER2 ECD. Its antitumor activity has not been completely ascertained, however, it is thought to result from a combination of antibody-dependent cell-mediated cytotoxicity, inhibition of cleavage of the ECD of the HER2 (8), decreased DNA repair, decreased intracellular signal transduction and 
anti-angiogenic effects $(9,10)$. Trastuzumab-based treatment strategy has established a milestone in the therapy of HER2-positive breast cancer with attractive clinical benefits in the treatment of metastatic breast cancer, as well as adjuvant chemotherapy and neoadjuvant chemotherapy.

Adding trastuzumab to chemotherapy in the first-line treatment of HER2-positive metastatic breast cancer (MBC) was based on the pivotal phase III trial in which 469 women with HER2-positive MBC were randomized to receive standard chemotherapy (paclitaxel or anthracycline/cyclophosphamide) with or without trastuzumab. The combination improved response rates (RRs; 50 vs. 32\%), extended time to progression (TTP; 7.4 vs. 4.6 months) and median overall survival (OS; 25 vs. 20 months) (11). Subsequently, results of two randomized trials demonstrated the benefit of adding trastuzumab to chemotherapy in the treatment of HER2-positive MBC, as well as significant improvements in TTP and OS $(12,13)$. Besides paclitaxel and docetaxel, other combination regimens of trastuzumab with chemotherapy drugs such as vinorelbine, capecitabine, platinum salts and gemcitabine, have also shown clinical benefit in patients with HER2-positive MBC (14-18). Single agent trastuzumab has also been evaluated, however, it yielded lower RRs compared with the combination of chemotherapy and trastuzumab (19). Thus, it seems appropriate to prescribe trastuzumab monotherapy for elderly or frail patients whose performance status permits administration of trastuzumab but not chemotherapy.

In the phase III TAnDEM post-menopausal patients with HER2-positive, oestrogen receptor (ER) + MBC were randomized into the primary therapy with anastrozole alone group or the anastrozole combined with trastuzumab. Results of that study showed that the addition of trastuzumab significantly improved RRs (20.3 vs. 6.8\%) and progression-free survival (PFS; 4.8 vs. 2.4 months) (20). Therefore, blockade of the downstream signaling pathway of the HER2 receptor might enhance endocrine sensitivity of HER2-positive, $\mathrm{ER}^{+}$breast cancer cells.

Trastuzumab has a marked effect on HER2-positive MBC, however, progression remains unavoidable. The synergy between trastuzumab and a variety of chemotherapeutic agents is not only exploited in first-line setting but also beyond disease progression in pretreated patients. Data from retrospective and prospective clinical studies suggest that continuation of trastuzumab with second- and third-line chemotherapeutic agents following disease progression on previous administration of trastuzumab is capable of eliciting objective responses and delaying disease progression (21-23). The Trastuzumab Beyond Progression study randomly assigned 156 patients who had progressed from trastuzumab to capecitabine with trastuzumab or capecitabine alone. Continuation of trastuzumab with capecitabine resulted in a significantly improved TTP (8.2 vs. 5.6 months) and RR (48.1 vs. $27.0 \%$ ) as compared with capecitabine alone (23). In the clinic, continued use of trastuzumab has been a therapeutic option for patients who progress during or after treatment with trastuzumab-containing treatment regimens.

After its approval in the metastatic setting, trastuzumab was evaluated in the adjuvant setting in several large prospective randomized trials. Results of a recent meta-analysis of published prospective randomized trials that included over
13,000 patients demonstrated that the combination of trastuzumab with adjuvant chemotherapy produced a significant benefit in disease-free survival (DFS) odds ratio $(\mathrm{OR})=0.69$, OS $(\mathrm{OR}=0.78)$, locoregional recurrence $(\mathrm{OR}=0.53)$ and distant recurrence $(\mathrm{OR}=0.62)$, as compared with chemotherapy alone (24). Outcome was significantly improved with trastuzumab as monotherapy following the completion of chemotherapy, and in combination with paclitaxel or docetaxel following the completion of doxorubicin plus cyclophosphamide, or given concurrently with carboplatin and docetaxel. Based on these results, adjuvant treatment of early-stage breast cancer with combined trastuzumab and chemotherapy has become standard in patients with HER2-positive tumors $>1 \mathrm{~cm}$ in size or positive lymph nodes. Few patients with HER2-positive tumors, a diameter of $\leq 1 \mathrm{~cm}$ and negative lymph nodes were included in the majority of randomized trials, suggesting that exhaustive data on trastuzumab for small node-negative tumors are lacking. Current data suggest that node-negative HER2-positive tumors of 0.6-1.0 $\mathrm{cm}$ are thought to benefit from adjuvant trastuzumab therapy, based on the evidence for an inferior clinical outcome in these patients (25). Two ongoing trials that include patients with small HER2-positive, node-negative breast tumors may explore less toxic chemotherapy and/or short-course trastuzumab. Currently, treatment with trastuzumab for 1 year in addition to chemotherapy is the only approved HER2-specific adjuvant treatment for patients with HER2-positive early breast cancer. Several trials have addressed the optimal duration of trastuzumab on adjuvant therapy. Recently, data from the HERA study, which is the only randomized trial investigating whether a longer duration of trastuzumab further improves efficacy outcome, suggested that OS in the 2-year and 1-year arm of trastuzumab was comparable [hazard ratio $1.05,95 \%$ confidence interval (CI), 0.86-1.28; $\mathrm{p}=0.63]$, but the cardiotoxicity was higher in the 2-year arm (7.2 vs. $4.1 \%)$. These results confirm that 1 year of adjuvant trastuzumab remains the standard of care for HER2-positive early breast cancer and the significant improvement in DFS and OS for trastuzumab persists at a median follow-up of 8 years (26). Findings of the NCCTG N9831 trial suggest that trastuzumab is more effective when initiated concurrently with the taxane component of adjuvant chemotherapy compared with initiation after completion of chemotherapy (27). This approach also reduces the duration of intravenous therapy by approximately 3 months, which might improve convenience (28).

Benefits from use of trastuzumab in HER2-positive early and $\mathrm{MBC}$ have been noted. However, the addition of trastuzumab to neoadjuvant treatment in the locally advanced setting is also attractive. Initial data on trastuzumab in the neoadjuvant setting revealed that the pathologic complete response $(\mathrm{pCR})$ rate, which is connected with a significantly improved outcome, increased from 26.0 to $65.2 \%$ with the addition of trastuzumab to sequential anthracycline- and taxane-based chemotherapy and long-term follow-up of the patients revealed a significantly lower relapse rate in patients receiving chemotherapy plus trastuzumab (29). Subsequently, several randomized trials evaluating the efficacy of a wide variety of trastuzumab-containing regimens in the neoadjuvant setting have been reported. A meta-analysis of randomized trials indicated that the addition of trastuzumab to neoad- 
juvant chemotherapy significantly improves $\mathrm{pCR}$ rates and event-free survival in patients with locally advanced breast cancer compared with chemotherapy alone (30). Therefore, the addition of trastuzumab to neoadjuvant chemotherapy in HER2-positive breast cancer is considered a standard option. In almost all trials, trastuzumab treatment was completed after surgery for a total duration of 1 year and this is currently recommended in the NCCN Clinical Practice guidelines (31).

In a large clinical trial, it was reported that trastuzumab is generally well tolerated despite an association with potential cardiotoxicity (32). Trastuzumab-related cardiac dysfunction, which involves asymptomatic decrease in the left ventricular ejection fraction (LVEF), may be influenced by concurrent or sequential chemotherapy as well as type of chemotherapy. A retrospective review of records for patients with $\mathrm{MBC}$ revealed that the highest incidence of cardiac dysfunction was observed with the concurrent use of trastuzumab and anthracycline (27\%) and the risk was substantially lower with trastuzumab and paclitaxel (13\%) or with trastuzumab alone (3-7\%) (33). A pivotal trial of trastuzumab plus chemotherapy in MBC did not identify any unexpected adverse events with the use of long-term (up to $40+$ months) trastuzumab (22). On the basis of available data on the use of trastuzumab in the adjuvant setting, cardiotoxicity seems to be treatable and mostly reversible and the risk of severe cardiac dysfunction ranges from 0.6 to $3.9 \%$ (32). Adjuvant trastuzumab trials have led to the introduction of rules for cardiac monitoring and the cessation of therapy $(34,35)$. The incidence of cardiac dysfunction was $0-9.4 \%$ experiencing a decrease of $>10 \%$ in LVEF when trastuzumab was added to neoadjuvant chemotherapy $(36,37)$. In contrast to the early experience in $\mathrm{MBC}$, concurrent neoadjuvant treatment with anthracycline-based regimen and trastuzumab appears to have an acceptable cardiac toxicity profile. Clinical evaluation prior to treatment with trastuzumab and anthracyclines in the neoadjuvant setting should include careful screening for cardiac risk factors (i.e., pre-existing cardiac diseases, baseline LVEF $50-55 \%$ in patients $>65$ years with hypertension, diabetes, or smoking habit and with BMI $>25$ ) and restriction of the cumulative anthracycline dose. Trastuzumab must be avoided if baseline LVEF is $<50 \%$.

Marked improvement has been observed in patients with HER2-positive breast cancer since the widespread use of trastuzumab; however, approximately $10 \%$ of patients develop a distant recurrence following adjuvant trastuzumab-based chemotherapy and all patients with MBC eventually develop disease progression (38). Furthermore, the risk of cardiotoxicity currently precludes certain patients from trastuzumab treatment, limiting the choice of agents that can be used concurrently with trastuzumab. Therefore, the development of novel targeted agents for use in HER2-positive breast cancer remains clinically significant.

Pertuzumab. Pertuzumab (Omnitarg; Genentech/Roche, South San Francisco, CA, USA) is an investigational fully humanized monoclonal antibody that targets the ECD of HER2 at a different site to trastuzumab and is able to inhibit ligand-induced homo- and hetero-dimerization of HER2 with other EGFR family members, including HER1, HER3 and HER4 $(39,40)$. Early clinical data showing only modest activity as a single agent (41) and preclinical data showing enhanced antitumor activity for the combination of pertuzumab plus trastuzumab (42) have led investigators to focus on future clinical studies of pertuzumab in combination with trastuzumab. In a phase II trial evaluating the combination of pertuzumab and trastuzumab, a $24.2 \% \mathrm{RR}$ and a $50 \%$ clinical benefit rate in 66 patients with HER2-positive MBC that progressed after trastuzumab-based therapy was reported (43). The promising results strongly demonstrate that pertuzumab can partially reverse trastuzumab resistance and the combination of the two antibodies can result in a synergistic efficacy. Combinations of pertuzumab and trastuzumab with chemotherapy have also being evaluated. A recently published clinical study that randomly assigned 808 patients with HER2-positive MBC to receive trastuzumab plus docetaxel with or without the combination of pertuzumab as first-line treatment demonstrated that the combination of pertuzumab plus trastuzumab plus docetaxel significantly prolonged PFS as compared with placebo plus trastuzumab plus docetaxel (18.5 vs. 12.4 months), and the interim analysis of OS showed a strong trend in favor of pertuzumab plus trastuzumab plus docetaxel (44). Pertuzumab has been approved by the US Food and Drug Administration in the first-line treatment of HER2-positive MBC (45). In a phase II randomized study of neoadjuvant setting, this dual-targeted drug combination plus docetaxel resulted in a statistically significant increase in pCR as compared with trastuzumab or pertuzumab plus docetaxel (45.8 vs. 29.0 or $24.0 \%$ ), and a pCR rate of $16.8 \%$ in patients with targeted therapy only (no chemotherapy) (46). Recent findings suggest the addition of pertuzumab to trastuzumab in HER2-positive breast cancer is a therapeutic option in the neoadjuvant setting (47). At present, there are ongoing studies with pertuzumab in the adjuvant setting.

Since trastuzumab and pertuzumab are structurally similar and target HER2, additive toxicity is expected when the two drugs are administered concurrently. The tolerability of pertuzumab in combination with trastuzumab have being evaluated in several randomized trials in patients with HER2-positive breast cancer. In a phase II trial of 66 patients, cardiotoxicity was less of an issue with only three patients experiencing an asymptomatic LVEF decline of $\geq 10 \%$ and an absolute LVEF of $<50 \%$, and there were no withdrawals due to cardiac-related events (43). A recently published clinical study also demonstrated that the combination of pertuzumab plus trastuzumab plus docetaxel did not increase cardiac toxic effects (44). In general, the combination of pertuzumab and trastuzumab was well tolerated. However, the data on cardiac safety with pertuzumab should be interpreted with caution as the trials were conducted in carefully selected patients.

Trastuzumab-DM1. Trastuzumab-DM1 (T-DM1; Genentech/Roche, South San Francisco, CA, USA) is a novel chemistry-driven antibody-drug conjugate combining trastuzumab with a potent antimicrotubule agent, DM1 (a derivate of the cytotoxic chemotherapy agent maytansine). This molecule is targeted to HER2-positive cancer cells and releases DM1, thereby inhibiting the assembly of cellular microtubules (48). In trastuzumab-DM1, trastuzumab, not only retains the known mechanisms of action of trastuzumab, but also acts as a carrier that delivers DM1 to the tumor cells labelled with HER2, rendering DM1 less toxic and more effective (49). In a phase II 
study of 110 patients with heavily pretreated HER2-positive MBC, trastuzumab-DM1 demonstrated that single-agent activity yielded a RR of $41.3 \%$ and PFS of 7.3 months in patients with HER2-positive MBC who had previously received the two HER2-directed therapies and multiple chemotherapeutic agents (50). A randomised phase II trial of trastuzumab-DM1 vs. trastuzumab/docetaxel in first-line, HER2-positive MBC demonstrated significant increases in RR (47.8 vs. 41.4\%) and PFS (14.2 vs. 9.2 months) with trastuzumab-DM1 compared with the control arm, respectively $(51,52)$. A phase Ib/II study evaluating the combination of trastuzumab-DM1 and pertuzumab in patients with previously untreated and relapsed HER2-positive MBC yielded a RR of $57.1 \%$ in previously untreated patients and a RR of 34.8\% in relapsed patients (53). Data from the ongoing phase III trials of trastuzumab-DM1 and combination with various agents remain to be reported.

Toxicities of trastuzumab-DM1 were mild and reversible, and included thrombocytopenia, elevated transaminases, fatigue, nausea, and anemia (49). No dose-limiting cardiotoxicity was observed (50).

\section{Tyrosine kinase inhibitors}

Lapatinib. Lapatinib (Tykerb; GlaxoSmithKline, London, UK) is a dual, orally administered small molecule tyrosine kinase inhibitor of HER1 and HER2, which binds reversibly to the intracellular ATP-binding pocket of the two receptors and inhibits receptor autophosphorylation, preventing the activation of downstream cellular signals that promote tumor cell survival and proliferation $(54,55)$. Lapatinib was approved in combination with capecitabine for the treatment of advanced or metastatic HER2-positive breast cancer based on the pivotal phase III trial in which 324 patients pretreated with an anthracycline, taxane and trastuzumab were randomized to receive capecitabine plus lapatinib or capecitabine alone (56). This trial showed that the addition of lapatinib to capecitabine significantly increased TTP (8.4 vs. 4.4 months) vs. capecitabine alone (56). The EGF30001 phase III trial which compared lapatinib plus paclitaxel with paclitaxel alone in the first-line setting demonstrated that treatment with paclitaxel plus lapatinib resulted in statistically significant improvements compared with paclitaxel alone in TTP (36.4 vs. 25.1 weeks), event-free survival (35.1 vs. 21.9 weeks) and RR (63.3 vs. 37.8\%) (57). In addition to trials using lapatinib plus capecitabine or paclitaxel combination therapy, clinical trials of lapatinib and other chemotherapy agents, such as nab-paclitaxel, cisplatin and gemcitabine, have also exhibited positive results $(58,59)$. In the EGF30008 phase III trial, which compared lapatinib plus letrozole with letrozole alone in treatment-naïve post-menopausal patients with hormone receptor-positive $\mathrm{MBC}$, a 5.2 month improvement in PFS was evident in the lapatinib plus letrozole arm (8.2 vs. 3.0 months) (60). Dual HER2 blockade with lapatinib and trastuzumab was also assessed in a phase III trial of lapatinib plus trastuzumab comparing lapatinib alone in patients with HER2-positive MBC whose disease had progressed on trastuzumab. The trial demonstrated a significant 4.5 month median OS improvement with lapatinib plus trastuzumab compared with lapatinib alone and support dual HER2 blockade, thus offering a chemotherapy-free option for patients with heavily pretreated HER2-positive MBC (61). Lapatinib monotherapy yielded a RR of $24 \%$ as first-line treatment (62) and a RR of $5.1 \%$ in heavily pretreated patients (63).

The brain is a common metastatic site for HER2-positive breast cancer. Results of one study suggest that almost $50 \%$ of patients treated with trastuzumab develop brain metastases (64). However, poor results of systemic treatment of brain metastases in HER2-positive breast cancer have been obtained, likely due to the inability of systemic therapies, including trastuzumab, to cross the blood-brain barrier. Although lapatinib is a small molecular compound which may penetrate the blood-brain barrier easily and achieve an effective concentration level in the cerebrospinal fluid, it may also control brain metastasis. A potential role for lapatinib in reducing brain metastases became apparent in an exploratory analysis of data from a phase III trial of lapatinib plus capecitabine $(56,65)$. The analysis demonstrated that lapatinib plus capecitabine treatment was associated with a lower rate of brain tumor progression, compared with capecitabine alone (65). This finding led to several trials of patients with brain metastases treated with lapatinib, with the results suggesting that lapatinib plays a role in the prevention and management of brain metastases in patients with HER2-positive breast cancer (66-68).

While the clinical evidence supported the use of lapatinib in the metastatic settings, lapatinib was evaluated in the neoadjuvant setting in several prospective randomized trials. A meta-analysis of randomized trials that compared the addition of lapatinib vs. trastuzumab or their combination to neoadjuvant chemotherapy in HER2-positive breast cancer showed that a significant increase of $\mathrm{pCR}$ rate was observed in the chemotherapy plus trastuzumab and lapatinib arm compared with chemotherapy plus trastuzumab [risk ratio of $1.39,95 \% \mathrm{CI}, 1.20-1.63 ; \mathrm{P}<0.001]$, pCR rate was higher in the chemotherapy plus trastuzumab arm vs. chemotherapy plus lapatinib (risk ratio of 1.25, 95\% CI, 1.08-1.43; $\mathrm{P}=0.003$ ), and grade III-IV toxicities were statistically more frequent in patients receiving chemotherapy plus lapatinib (69). These data added further evidence supporting the superiority of a dual-HER2 inhibition for the treatment of HER2-positive breast cancer. The direct comparison of trastuzumab and lapatinib showed that lapatinib is inferior in terms of pCR and associated with a higher risk for toxicity in the neoadjuvant setting. Thus, use of lapatinib in the neoadjuvant setting should be considered experimental. In addition to trials in the neoadjuvant setting, several adjuvant studies of lapatinib are ongoing. In the future, these trials should establish whether lapatinib and trastuzumab are to be used together or sequentially, and which settings are optimal for the two agents.

The systemic analysis of lapatinib demonstrated that it is well tolerated with manageable toxic effects (70). Although the same pathway was targeted, no increase in symptomatic cardiac events with the addition of lapatinib occurred, and the incidence of cardiac toxicity was lower with lapatinib compared with trastuzumab. Grade 3/4 treatment-related toxicities of lapatinib are uncommon with grade 3 diarrhea reported in $7 \%$ and all other toxicities (not specified) in $<3 \%$ of patients.

Neratinib. Neratinib (HKI-272; Pfizer, New York, NY, USA) is an irreversible, orally administered small molecule TKI of 
HER1, HER2 and HER4 that covalently binds to the cysteine residues of the ATP-binding portion of the HER TKs (71). An open-label, phase II multicenter trial of single-agent neratinib in 136 patients with HER2-positive MBC showed a RR of $24 \%$ in patients previously treated with trastuzumab, and a RR of $56 \%$ in trastuzumab-naïve patients. PFS at 16 weeks was 59 and $78 \%$, respectively (72). No grade 3 or 4 cardiotoxicity related to neratinib was reported, but grade 3 and 4 diarrhea was the most frequently occurring adverse effect. In combination with vinorelbine in patients pretreated with trastuzumab, RRs of 41 and $8 \%$ were observed in those who had not and had received prior lapatinib, respectively (73). The potential combinations of neratinib with various chemotherapy agents, trastuzumab, lapatinib and new targeted agents in the metastatic, adjuvant and even neoadjuvant settings are under investigation. These results and future perspectives remain to be elucidated and reported.

Afatinib. Afatinib (BIBW 2992; Boehringer Ingelheim, Ingelheim, Germany) is a novel, oral, small-molecule TKI that covalently binds and irreversibly blocks all kinase-competent HER family members (74). In an open-label, single-arm phase II study which explored afatinib activity in 41 HER2-positive breast cancer patients progressing after trastuzumab treatment, 4 patients had partial responses and 15 patients had stable disease. Median PFS was 15.1 weeks, and median OS was 61.0 weeks. The most frequently occurring grade 3 treatment-related adverse events were diarrhea $(24.4 \%)$ and rash $(9.8 \%)$. Afatinib monotherapy had promising clinical activity in heavily pretreated HER2-positive breast cancer patients following progression on trastuzumab (75). An ongoing global phase III study is evaluating afatinib plus vinorelbine versus trastuzumab plus vinorelbine in patients with HER2-positive MBC progressing after trastuzumab treatment. Afatinib is also under investigation in various combinations and in the neoadjuvant setting.

\section{Conclusion}

Treatment specifically targeted at HER2 has improved survival during the past decade in patients with HER2-positive breast cancer. Nevertheless, resistance remains a challenge, particularly in the metastatic setting. With the deepening fundamental understanding of molecular correlations and characterization of breast cancer, new agents are in clinical development, including those directed at the HER2 receptor itself and those targeting downstream effectors and interacting compensatory signaling pathways such as hsp90, mTOR and IGF-1R inhibitors. Such results are likely to useful in the prognotic effect of HER2-positive breast cancer.

\section{References}

1. Perou CM, Sorlie T, Eisen MB, van de Rijn M, Jeffrey SS, Rees CA, Pollack JR, Ross DT, Johnsen H, Akslen LA, et al: Molecular portraits of human breast tumors. Nature 406: 747-752, 2000.

2. Slamon DJ, Clark GM, Wong SG, Levin WJ, Ullrich A and McGuire WL: Human breast cancer: correlation of relapse and survival with amplification of the HER-2/neu oncogene. Science 235: 177-182, 1987.
3. Slamon DJ, Godolphin W, Jones LA, Holt JA, Wong SG Keith DE, Levin WJ, Stuart SG, Udove J, Ullrich A, et al: Studies of the HER-2/neu proto-oncogene in human breast and ovarian cancer. Science 244: 707-712, 1989.

4. Yarden Y and Sliwkowski MX: Untangling the ErbB signalling network. Nat Rev Mol Cell Biol 2: 127-137, 2001.

5. Klapper LN, Kirschbaum MH, Sela M and Yarden Y: Biochemical and clinical implications of the ErbB/HER signaling network of growth factor receptors. Adv Cancer Res 77: 25-79, 2000.

6. Hynes NE and Lane HA: ERBB receptors and cancer: the complexity of targeted inhibitors. Nat Rev Cancer 5: 341-354, 2005.

7. Graus-Porta D, Beerli RR, Daly JM and Hynes NE: ErbB-2, the preferred heterodimerization partner of all ErbB receptors, is a mediator of lateral signaling. EMBO J 16: 1647-1655, 1997.

8. Molina MA, Codony-Servat J, Albanell J, Rojo F, Arribas J and Baselga J: Trastuzumab (herceptin), a humanized anti-Her2 receptor monoclonal antibody, inhibits basal and activated Her2 ectodomain cleavage in breast cancer cells. Cancer Res 61: 4744-4749, 2001.

9. Baselga J, Albanell J, Molina MA and Arribas J: Mechanism of action of trastuzumab and scientific update. Semin Oncol 28 (5 Suppl 16): 4-11, 2001

10. Spector NL and Blackwell KL: Understanding the mechanisms behind trastuzumab therapy for human epidermal growth factor receptor 2-positive breast cancer. J Clin Oncol 27: 5838-5847, 2009.

11. Slamon DJ, Leyland-Jones B, Shak S, Fuchs H, Paton V, Baiamonde A, Fleming T, Eiermann W, Wolter J, Pegram M, et al: Use of chemotherapy plus a monoclonal antibody against HER2 for metastatic breast cancer that overexpresses HER2. N Engl J Med 344: 783-792, 2001.

12. Marty M, Cognetti F, Maraninchi D, Snyder R, Mauriac L, Tubiana-Hulin M, Chan S, Grimes D, Antón A, Lluch A, et al: Randomized phase II trial of the efficacy and safety of trastuzumab combined with docetaxel in patients with human epidermal growth factor receptor 2-positive metastatic breast cancer administered as first-line treatment: the M77001 study group. J Clin Oncol 23: 4265-4274, 2005.

13. Gasparini G, Gion M, Mariani L, Papaldo P, Crivellari D, Filippelli G, Morabito A, Silingardi V, Torino F, Spada A, et al: Randomized phase II trial of weekly paclitaxel alone versus trastuzumab plus weekly paclitaxel as first-line therapy of patients with Her-2 positive advanced breast cancer. Breast Cancer Res Treat 101: 355-365, 2007.

14. Burstein HJ, Keshaviah A, Baron AD, Hart RD, Lambert-Falls R, Marcom PK, Gelman R and Winer EP: Trastuzumab plus vinorelbine or taxane chemotherapy for HER2-overexpressing metastatic breast cancer: the trastuzumab and vinorelbine or taxane study. Cancer 110: 965-972, 2007.

15. Yamamoto D, Iwase S, Kitamura K, Odagiri H, Yamamoto C and Naqumo Y: A phase II study of trastuzumab and capecitabine for patients with HER2-overexpressing metastatic breast cancer: Japan Breast Cancer Research Network (JBCRN) 00 Trial. Cancer Chemother Pharmacol 61: 509-514, 2008.

16. Pegram MD, Lipton A, Hayes DF, Weber BL, Baselga JM, Tripathy D, Baly D, Bauqhman SA, Twaddell T, Glaspy JA and Slamon DJ: Phase II study of receptor-enhanced chemosensitivity using recombinant humanized anti-p185HER $2 /$ neu monoclonal antibody plus cisplatin in patients with HER2/neu-overexpressing metastatic breast cancer refractory to chemotherapy treatment. J Clin Oncol 16: 2659-2671, 1998.

17. Pegram MD, Pienkowski T, Northfelt DW, Eiermann W, Patel R, Fumoleau P, Quan E, Crown J, Toppmeyer D, Smylie M, et al: Results of two open-label, multicenter phase II studies of docetaxel, platinum salts, and trastuzumab in HER2-positive advanced breast cancer. J Natl Cancer Inst 96: 759-769, 2004.

18. Yardley DA, Burris HA 3rd, Simons L, Spigel DR, Greco FA, Barton JH, Shipley D, Drosick D and Hainsworth JD: A phase II trial of gemcitabine/carboplatin with or without trastuzumab in the first-line treatment of patients with metastatic breast cancer. Clin Breast Cancer 8: 425-431, 2008.

19. Vogel CL, Cobleigh MA, Tripathy D, Gutheil JC, Harris LN Fehrenbacher L, Slamon DJ, Murphy M, Novotny WF, Burchmore M, et al: Efficacy and safety of trastuzumab as a single agent in first-line treatment of HER2-overexpressing metastatic breast cancer. J Clin Oncol 20: 719-726, 2002. 
20. Kaufman B, Mackey JR, Clemens MR, Bapsy PP, Vaid A, Wardley A, Tjulandin S, Jahn M, Lehle M, Feyereislova A, et al: Trastuzumab plus anastrozole versus anastrozole alone for the treatment of postmenopausal women with human epidermal growth factor receptor 2-positive, hormone receptor-positive metastatic breast cancer: results from the randomized phase III TAnDEM study. J Clin Oncol 27: 5529-5537, 2009.

21. Gelmon KA, Mackey J, Verma S, Gertler SZ, Bangemann N, Klimo P, Schneeweiss A, Bremer K, Soulieres D, Tonkin K, et al: Use of trastuzumab beyond disease progression: observations from a retrospective review of case histories. Clin Breast Cancer 5: 52-62, 2004.

22. Tripathy D, Slamon DJ, Cobleigh M, Arnold A, Saleh M, Mortimer JE, Murphy M and Stewart SJ: Safety of treatment of metastatic breast cancer with trastuzumab beyond disease progression. J Clin Oncol 22: 1063-1070, 2004.

23. Von Minckwitz G, du Bois A, Schmidt M, Maass N, Cufer T, de Jongh FE, Maartense E, Zielinski C, Kaufmann M, Bauer W, et al: Trastuzumab beyond progression in human epidermal growth factor receptor 2-positive advanced breast cancer: a german breast group 26/breast international group 03-05 study. J Clin Oncol 27: 1999-2006, 2009.

24. Yin W, Jiang Y, Shen Z, Shao Z and Lu J: Trastuzumab in the adjuvant treatment of HER2-positive early breast cancer patients: a meta-analysis of published randomized controlled trials. PLoS One 6: e21030, 2011.

25. Joerger M, Thurlimann B and Huober J: Small HER2-positive, node-negative breast cancer: who should receive systemic adjuvant treatment? Ann Oncol 22: 17-23, 2011.

26. Goldhirsch A, Piccart-Gebhart MJ, Procter M, de Azambuja E, Weber HA, Untch M, Smith I, Gianni L, Jackisch C, Cameron D, Bell R, Dowsett M, et al: HERA TRIAL: 2 years versus 1 year of trastuzumab after adjuvant chemotherapy in women with HER2-positive early breast cancer at 8 years of median follow up. Presented at: 35th Annual San Antonio Breast Cancer Symposium; December 4-8, 2012; San Antonio, TX. abs. S5-2

27. Perez EA, Dueck AC, Press MF, Jenkins RB, Kim C, Chen B, Villalobos I, Paik S, Buyse M, Wiktor AE, et al: Results of chemotherapy alone, with sequential or concurrent addition of 52 weeks of trastuzumab in the NCCTG N9831 HER2-positive adjuvant breast cancer trial. Cancer Res 70: a5640, 2009.

28. Mackey J, Mcleod D, Ragaz J, Gelmon K, Verma S, Pritchard K, Laing K, Provencher L, and Charbonneau LF: Adjuvant targeted therapy in early breast cancer. Cancer 115: 1154-1168, 2009.

29. Buzdar AU, Ibrahim NK, Francis D, Booser DJ, Thomas ES, Theriault RL, Pusztai L, Green MC, Arun BK, Giordano SH, et al: Significantly higher pathologic complete remission rate after neoadjuvant therapy with trastuzumab, paclitaxel, and epirubicin chemotherapy : results of a randomized trial in human epidermal growth factor receptor 2-positive operable breast cancer. J Clin Oncol 23: 3676-3685, 2005.

30. Valachis A, Mauri D, Polyzos NP, Chlouverakis G, Mavroudis D and Georgoulias V: Trastuzumab combined to neoadjuvant chemotherapy in patients with HER2-positive breast cancer: a systematic review and meta-analysis. Breast 20: 485-490, 2011.

31. NCCN Clinical Practice Guidelines in Oncology: Breast Cancer. Version 1.2013. Available at www.NCCN.com: Accessed February 22, 2013

32. Garnock-Jones KP, Keating GM and Scott LJ: Trastuzumab: a review of its use as adjuvant treatment in human epidermal growth factor receptor 2 (HER2)-positive early breast cancer. Drugs 70: 215-239, 2010

33. Seidman A, Hudis C, Pierri MK, Shak S, Paton V, Ashby M, Murphy M, Stewart SJ and Keefe D: Cardiac dysfunction in the trastuzumab clinical trials experience. J Clin Oncol 20: $1215-1221,2002$

34. Rastogi P, Jeong J, Geyer CE, Romond EH, Swain SM, Ewer MS, Rathi V, Fehrenbacher L, Brufsky A, Azar CA, et al: Five-year update of cardiac dysfunction on NSABP B-31, a randomized trial of sequential doxorubicin/cyclophosphamide $(\mathrm{AC}) \rightarrow$ paclitaxel (T) compared to $\mathrm{AC} \rightarrow \mathrm{T}$ with trastuzumab $(\mathrm{H})$. J Clin Oncol 25 6s. abs. LBA513, 2007.

35. Perez EA, Suman VJ, Davidson NE, Sledge GW, Kaufman PA, Hudis CA, Martino S, Gralow JR, Dakhil SR, Ingle JN, et al: Cardiac safety analysis of doxorubicin and cyclophosphamide followed by paclitaxel with or without trastuzumab in the North Central Cancer Treatment Group N9831 adjuvant breast cancer trial. J Clin Oncol 26: 1231-1238, 2008.
36. Buzdar AU, Valero V, Ibrahim NK, Francis D, Broglio KR, Theriault RL, Pusztai L, Green MC, Singletary SE, Hunt KK, et al: Neoadjuvant therapy with paclitaxel followed by 5 -fluorouracil, epirubicin, and cyclophosphamide chemotherapy and concurrent trastuzumab in human epidermal growth factor receptor 2-positive operable breast cancer: an update of the initial randomized study population and data of additional patients treated with the same regimen. Clin Cancer Res 13: 228-233, 2007.

37. Kelly H, Kimmick G, Dees EC, Collichio F, Gatti L, Sawyer L, Ivanova A, Dressler L, Graham ML and Carey LA: Response and cardiac toxicity of trastuzumab given in conjunction with weekly paclitaxel after doxorubicin/cyclophosphamide. Clin Breast Cancer 7: 237-243, 2006.

38. Nahta R and O'Regan RM: Evolving strategies for overcoming resistance to HER2-directed therapy: targeting the PI3K/Akt/ mTOR pathway. Clin Breast Cancer 10: S72-S78, 2010.

39. Aqus DB, Akita RW, Fox WD, Lewis GD, Higgins B, Pisacane PI, Lofgren JA, Tindell C, Evans DP, Maiese K, et al: Targeting ligand-activated ErbB2 signaling inhibits breast and prostate tumor growth. Cancer Cell 2: 127-137, 2002.

40. Franklin MC, Carey KD, Vajdos FF, Leahy DJ, de Vos AM and Sliwkowski MX: Insights into ErbB signaling from the structure of the ErbB2-pertuzumab complex. Cancer Cell 5: 317-328, 2004.

41. Aqus DB, Gordon MS, Taylor C, Natale RB, Karlan B, Mendelson DS, Press MF, Allison DE, Sliwkowski MX, Lieberman G, et al: Phase I clinical study of pertuzumab, a novel HER dimerization inhibitor, in patients with advanced cancer. J Clin Oncol 23: 2534-2543, 2005.

42. Scheuer W, Friess T, Burtscher H, Bossenmaier B, Endl J and Hasmann M: Strongly enhanced antitumor activity of trastuzumab and pertuzumab combination treatment on HER2-positive human xenograft tumor models. Cancer Res 69: 9330-9336, 2009.

43. Baselga J, Gelmon KA, Verma S, Wardley A, Conte P, Miles D, Bianchi G, Cortes J, McNally VA, Ross GA, et al: Phase II trial of pertuzumab and trastuzumab in patients with human epidermal growth factor receptor 2-positive metastatic breast cancer that progressed during prior trastuzumab therapy. J Clin Oncol 28: $1138-1144,2010$.

44. Baselga J, Cortés J, Kim SB, Im SA, Hegg R, Im YH, Roman L, Pedrini JL, Pienkowski T, Knott A, et al: Pertuzumab plus trastuzumab plus docetaxel for metastatic breast cancer. N Engl J Med 366: 109-119, 2012.

45. No authors listed: FDA approves pertuzumab for breast cancer. Cancer Discov 2: OF6, 2012.

46. Gianni L, Pienkowski T, Im YH, Roman L, Tseng LM, Liu MC, Lluch A, Staroslawska E, de la Haba-Rodriguez J, Im SA, et al: Efficacy and safety of neoadjuvant pertuzumab and trastuzumab in women with locally advanced, inflammatory, or early HER2-positive breast cancer (NeoSphere): a randomised multicentre, open-label, phase 2 trial. Lancet Oncol 13: 25-32, 2012.

47. Von Minckwitz G, Loibl S and Untch M: What is the current standard of care for anti-HER2 neoadjuvant therapy in breast cancer? Oncology (Williston Park) 26: 20-26, 2012.

48. Lewis Phillips GD, Li G, Dugger DL, Crocker LM, Parsons KL, Mai E, Blattler WA, Lambert JM, et al: Targeting HER2-positive breast cancer with trastuzumab-DM1, an antibody-cytotoxic drug conjugate. Cancer Res 68: 9280-9290, 2008.

49. Krop IE, Beeram M, Modi S, Jones SF, Holden SN, Yu W, Girish S, Tibbitts J, Yi JH, Sliwkowski MX, et al: Phase I study of trastuzumab-DM1, an HER2 antibody-drug conjugate, given every 3 weeks to patients with HER2-positive metastatic breast cancer. J Clin Oncol 28: 2698-2704, 2010.

50. Krop IE, Lorusso P, Miller KD, Modi S, Yardley D, Rodriguez G, Guardino E, Lu M, Zheng M, Girish S, et al: A phase II study of trastuzumab emtansine in patients with human epidermal growth factor receptor 2-positive metastatic breast cancer who were previously treated with trastuzumab, lapatinib, an anthracycline, a taxane, and capecitabine. J Clin Oncol 30: 3234-3241, 2012.

51. Perez EA, Dirix L, Kocsis J, Bianchi GV, Lu J, Vinholes J, Guardino E, Song C, Tong B, Ng V, et al: Efficacy and safety of trastuzumab-DM1 vs trastuzumab plus docetaxel in HER2-positive metastatic breast cancer patients with no prior chemotherapy for metastatic disease: preliminary results of a randomized, multicenter, open-label phase 2 study (TDM4450G). Ann Oncol 21: abs. LBA3, 2010. 
52. Hurvitz S, Dirix L, Kocsis J, Bianchi GV, Lu J, Vinholes J, GuardinoE,Song C,Tong B, etal: Trastuzumabemtansine(T-DM1) vs trastuzumab plus docetaxel $(\mathrm{H}+\mathrm{T})$ in previously-untreated HER2-positive metastatic breast cancer (MBC): primary results of a randomized, multicenter, open-label phase II study (TDM4450g/B021976). Eur J Cancer 47: Abs. 5001, 2011

53. Dieras V, Harbeck $N$ and Albain K: A phase Ib/II trial of trastuzumab-DM1 with pertuzumab for patients with HER2-positive, locally advanced or metastatic breast cancer: interim efficacy and safety results. Presented at: 33rd Annual San Antonio Breast Cancer Symposium; December 8-12,2010; San Antonio, TX. abs. P3-14-01.

54. Medina PJ and Goodin S: Lapatinib: a dual inhibitor of human epidermal growth factor receptor tyrosine kinases. Clin Ther 30: $1426-1447,2008$

55. Tevaarwerk AJ and Kolesar JM: Lapatinib: a small-molecule inhibitor of epidermal growth factor receptor and human epidermal growth factor receptor-2 tyrosine kinases used in the treatment of breast cancer. Clin Ther 31: 2332-2348, 2009.

56. Geyer CE, Forster J, Lindquist D, Chan S, Romieu CG, Pienkowski T, Jagiello-Gruszfeld A, Crown J, Chan A, Kaufman B, et al: Lapatinib plus capecitabine for HER2-positive advanced breast cancer. N Engl J Med 355: 2733-2743, 2006.

57. Di Leo A, Gomez HL, AzizZ,Zvirbule Z, Bines J, Arbushites MC, Guerrera SF, Koehler M, Oliva C, Stein SH, et al: Phase III, double-blind, randomized study comparing lapatinib plus paclitaxel with placebo plus paclitaxel as first-line treatment for metastatic breast cancer. J Clin Oncol 26: 5544-5552, 2008.

58. Yardley DA, Hart L, Bosserman L, Salleh MN, Waterhouse DM, Hagan MK, Richards P, DeSilvio ML, Mahoney JM and Nagarwala Y: Phase II study evaluating lapatinib in combination with nab-paclitaxel in HER2-overexpressing metastatic breast cancer patients who have received no more than one prior chemotherapeutic regimen. Breast Cancer Res Treat 137: 457-464, 2013.

59. Valero M, Ruiz-Borrego $M$ and Salvador D: Cisplatin, gencitabine, and lapatinib in patients with HER2-positive metastatic breast cancer: an experience in routine clinical practice. ASCO Annual Meeting J Clin Oncol 29 abs. e11005, 2011.

60. Johnston S, Pippen J Jr, Pivot X, Lichinitser M, Sadeghi S, Dieras V, Gomez HL, Romieu G, Manikhas A, Kennedy MJ, et al: Lapatinib combined with letrozole versus letrozole and placebo as first-line therapy for postmenopausal hormone receptor-positive metastatic breast cancer. J Clin Oncol 27 5538-5546, 2009.

61. Blackwell KL, Burstein HJ, Storniolo AM, Rugo HS, Sledge G, Aktan G, Ellis C, Florance A, Vukelja S, Bischoff J, et al: Overall survival benefit with lapatinib in combination with trastuzumab for patients with human epidermal growth factor receptor 2-positive metastatic breast cancer: final results from the EGF104900 Study. J Clin Oncol 30: 2585-2592, 2012.

62. Gomez HL, Doval DC, Chavez MA, Ang PC, Aziz Z, Nag S, $\mathrm{Ng} \mathrm{C}$, Franco SX, Chow LW, Arbushites MC, et al: Efficacy and safety of lapatinib as first-line therapy for ErbB2-amplified locally advanced or metastatic breast cancer. J Clin Oncol 26: 2999-3005, 2008.

63. Blackwell KL, Pegram MD, Tan-Chiu E, Schwartzberg LS, Arbushites MC, Maltzman JD, Forster JK, Rubin SD, Stein SH and Burstein HJ: Single-agent lapatinib for HER2-overexpressing advanced or metastatic breast cancer that progressed on first- or secondline trastuzumab-containing regimens. Ann Oncol 20: 1026-1031, 2009.
64. Lai R, Dang CT, Malkin MG and Abrey LE: The risk of central nervous system metastases after trastuzumab therapy in patients with breast carcinoma. Cancer 101: 810-816, 2004

65. Cameron D, Casey M, Press M, Lindquist D, Pienkowski T, Romieu CG, Chan S, Jagiello-Gruszfeld A, Kaufman B, Crown J, et al: A phase III randomized comparison of lapatinib plus capecitabine versus capecitabine alone in women with advanced breast cancer that has progressed on trastuzumab: updated efficacy and biomarker analyses. Breast Cancer Res Treat 112: $533-543,2008$

66. Lin NU, Carey LA, Liu MC, Younger J, Come SE, Ewend M, Harris GJ, Bullitt E, Van den Abbeele AD, Henson JW, et al: Phase II trial of lapatinib for brain metastases in patients with human epidermal growth factor receptor 2-positive breast cancer. J Clin Oncol 26: 1993-1999, 2008.

67. Ro J, Park S, Kim S, Kim TY, Im YH, Rha SY, Chung JS, Moon H and Santillana S: Clinical outcomes of HER2-positive metastatic breast cancer patients with brain metastasis treated with lapatinib and capecitabine: an open-label expanded access study in Korea. BMC Cancer 12: 322, 2012.

68. Yap YS, Cornelio GH, Devi BC, Khorprasert C, Kim SB, Kim TY, Lee SC, Park YH, Sohn JH, Sutandyo N, et al: Brain metastases in Asian HER2-positive breast cancer patients: anti-HER2 treatments and their impact on survival. Br J Cancer 107: 1075-1082, 2012

69. Valachis A, Nearchou A, Lind P and Mauri D: Lapatinib, trastuzumab or the combination added to preoperative chemotherapy for breast cancer: a meta-analysis of randomized evidence. Breast Cancer Res Treat 135: 655-662, 2012.

70. Kopper L: Lapatinib: a sword with two edges. Pathol Oncol Res 14: 1-8, 2008.

71. Tsou HR, Overbeek-Klumpers EG, Hallett WA, Reich MF, Floyd MB, Johnson BD, Michalak RS, Nilakantan R, Discafani C, Golas J, et al: Optimization of 6,7-disubstituted-4-(arylamino) quinoline-3-carbonitriles as orally active, irreversible inhibitors of human epidermal growth factor receptor-2 kinase activity. J Med Chem 48: 1107-1131, 2005.

72. Burstein HJ, Sun Y, Dirix LY, Jiang Z, Paridaens R, Tan AR, Awada A, Ranade A, Jiao S, Schwartz G, et al: Neratinib, an irreversible ErbB receptor tyrosine kinase inhibitor, in patients with advanced ErbB2-positive breast cancer. J Clin Oncol 28: 1301-1307, 2010.

73. Awada A, Dirix L, Manso Sanchez L, Xu B, Luu T, Dieras V, Hershman DL, Agrapart V, Ananthakrishnan R and Staroslawska E: Safety and efficacy of neratinib (HKI-272) plus vinorelbine in the treatment of patients with ErbB2-positive metastatic breast cancer pretreated with anti-HER2 therapy. Ann Oncol 24: 109-116, 2013.

74. Li D, Ambrogio L, Shimamura T, Kubo S, Takahashi M, Chirieac LR, Padera RF, Shapiro GI, Baum A, Himmelsbach F, et al: BIBW2992, an irreversible EGFR/HER2 inhibitor highly effective in preclinical lung cancer models. Oncogene 27: 4702-4711, 2008

75. Lin NU, Winer EP, Wheatley D, Carey LA, Houston S, Mendelson D, Munster P, Frakes L, Kelly S, Garcia AA, et al: A phase II study of afatinib (BIBW 2992), an irreversible ErbB family blocker, in patients with HER2-positive metastatic breast cancer progressing after trastuzumab. Breast Cancer Res Treat 133: 1057-1065, 2012. 